\title{
Experimental Investigation of the Vibration and Noise and Structural Optimization of Motor
}

\author{
ZHONG Yu-hua' ${ }^{1}$, SUN Guo-hui ${ }^{2}$ \\ ${ }^{1}$ Guangzhou College of South China University of Technology, Guangzhou 510800,P.R.China \\ ${ }^{2}$ China Construction First Building(Group) Corporation Limited, Shenzhen518000,P.R.China \\ ayhua.zhong@gmail.com, ${ }^{b} 457219202 @ q q . c o m$
}

Keywords: Vibration, Acoustic noise, Motor, Resonance, Electromagnetic force

Abstract. Vibration and noise always occur simultaneously. Vibration is the source of noise. All noises are the transmission of the vibration energy of vibrating body on medium, whether solid or fluid. The noises may through many mediums to our ears and the noises may be very complicated, which are comprised by many vibration sources. However, it is easy to measure the magnitude of the noise. If we hope to reduce the noise, we should research the source of the vibration at first. The frequencies components of the noise can tell me which vibrating body with the maximum vibration. Because the noise component and it's vibration source have the same frequency. In this section, two motor have been tested. We have recorded the magnitude of the noise. Finally, we carried out spectrum analysis for the test results. Based on the results of motor natural frequencies through finite element analysis, we found, the noise is may caused by the resonance of some parts of the motor when their natural frequency equal to the frequency of the electromagnetic force. We can modify the structure of the motor to reduce the noise.

\section{Test objective}

At first, the noise level should be measured. Based on the test data, the frequencies components of the noise can be determined. This data will be combined with the results of the finite element analysis about the natural frequencies of the motor to find which parts of the structure have been excited to produce resonance. In the end, the structural style of the motor will be optimized to avoid resonance and reduce the noise.

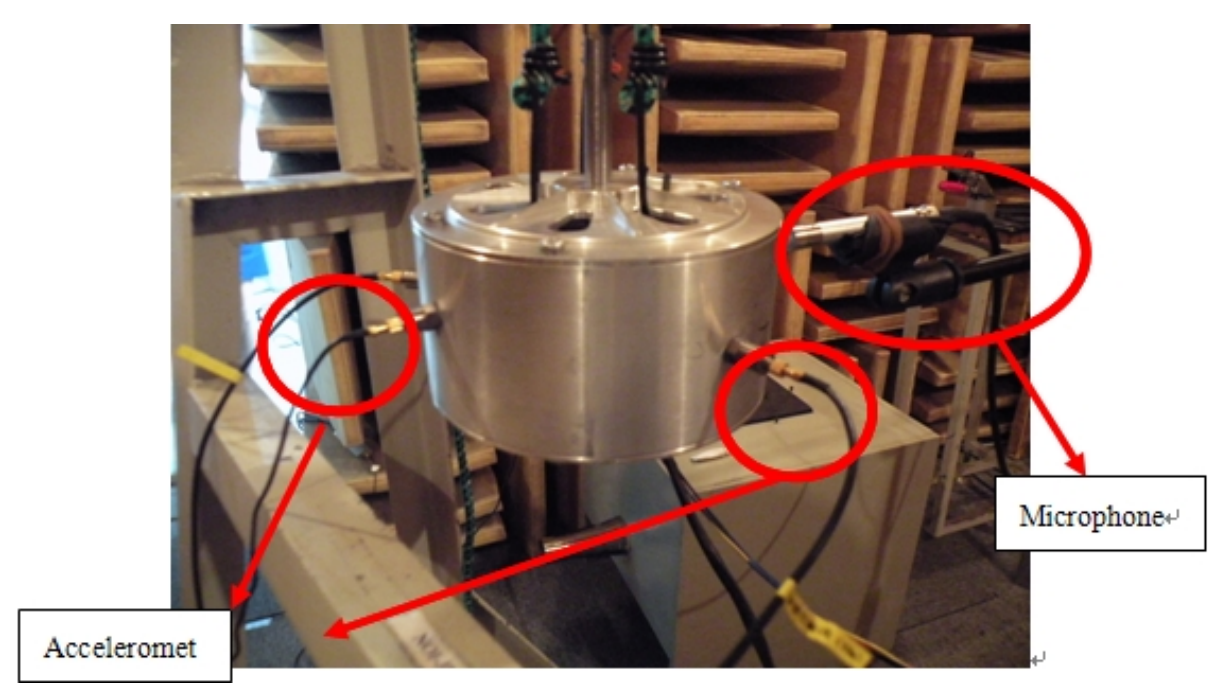

Fig. 1. Aluminum stator shell SRM 


\section{Testing process}

There are two motors, which hang freely to be tested in a semi-anechoic room, one with aluminum stator shell, another with gray cast iron stator shell, as showing in Fig.1. Four accelerometers have been attached with 502\# mucilage at points directly behind the appropriate stator poles and midway along the motor as are given in Fig.1.

The acoustic noise was measured using a microphone. In order to measure the acoustic noise which is induced by each excited stator pole, the microphone was located in line with the radial axis of one of stator poles and at one centimeter above the surface of the stator shell. When in experiment, the door of the semi-anechoic room was closed to prevent external disturbance, all according to the requirements of the standard noise experiment. In this experiment, the motor was set to various speeds to test the vibration and noise. The vibration accelerations signals of the stator shell at four points were detected by accelerometers. The signals were mechanical signals, they were transferred to electrical signals and sent to the dynamic signal analyzer to be changed to digital signals and in the end be shown on the computer.

\section{Experiments results}

In the experiments, we recorded the vibration accelerations, velocities and displacements of the motor at a series of speeds. In fact, we will take more attention to the acceleration and velocity of the motor, because the vibration is caused by the electromagnetic force, the force is the product of weight and acceleration. Usually, the weight is constant, if the acceleration is known, the force can be verified. Noises are the transmission of the vibration energy of vibrating body on medium. The vibration energy is decided by the vibrating velocity. So, the velocity is very important for the research on noise. The acceleration frequency-domain curve and velocity frequency-domain curve of the aluminum stator shell motor with the speeds of 3500 $/ \mathrm{min}, 6000 \mathrm{r} / \mathrm{min}$ and $9000 \mathrm{r} / \mathrm{min}$ are given in Fig.4 to Fig.6. On this curves, each peak value according to a resonance point. The acceleration frequency-domain curve, velocity frequency-domain curve and noise frequency-domain curve of the aluminum stator shell motor are given in Fig.3-Fig.9. From the experiment results we recorded, the vibration state of the gray cast iron stator shell motor is similar to the aluminum stator shell motor, so, we don't list the graphic analyses, only list relevant noise levels in table 2 and table 3. 
Table 1 Noise level of Aluminum stator shell motor

\begin{tabular}{|c|c|c|c|c|c|c|c|}
\hline \multicolumn{8}{|c|}{ Aluminum stator shell motor } \\
\hline \multirow{2}{*}{$2500 \mathrm{r} / \mathrm{min}$} & Frequency $(\mathrm{Hz})$ & 1364.1 & 2456.7 & 1910.4 & 1092.5 & 2731.3 & 1638.8 \\
\hline & Noise level(db) & 68.2 & 63.5 & 58.8 & 56.1 & 55.2 & 53 \\
\hline \multirow{2}{*}{$3500 \mathrm{r} / \mathrm{min}$} & Frequency $(\mathrm{Hz})$ & 2539.1 & 1074.2 & 1806.6 & 3271.5 & 1464.8 & 2880.9 \\
\hline & Noise level(db) & 67.3 & 64.5 & 62.2 & 54.9 & 54.1 & 52.9 \\
\hline \multirow{2}{*}{$4500 \mathrm{r} / \mathrm{min}$} & Frequency $(\mathrm{Hz})$ & 1416 & 1855.5 & 2343.8 & 1074.2 & 2783.2 & 3710.9 \\
\hline & Noise level(db) & 71.8 & 67.3 & 64.9 & 61.2 & 60.9 & 59.6 \\
\hline \multirow{2}{*}{$6000 \mathrm{r} / \mathrm{min}$} & Frequency $(\mathrm{Hz})$ & 1855.5 & 97.66 & 1220.7 & 1123 & 634.8 & 2490.23 \\
\hline & Noise level(db) & 81.55 & 69.1 & 67 & 66.56 & 66.1 & 66.1 \\
\hline \multirow{2}{*}{$8000 \mathrm{r} / \mathrm{min}$} & Frequency $(\mathrm{Hz})$ & 1074.2 & 830.1 & 8105.5 & 146.5 & 2441.4 & 4052.7 \\
\hline & Noise level(db) & 77.1 & 75.6 & 73.4 & 72.8 & 72.7 & 72.2 \\
\hline \multirow{2}{*}{$9000 \mathrm{r} / \mathrm{min}$} & Frequency $(\mathrm{Hz})$ & 1806.6 & 927.9 & 3613.3 & 9960.9 & 1074.2 & 146.5 \\
\hline & Noise level(db) & 88.1 & 78.8 & 74.4 & 73.4 & 73.4 & 73 \\
\hline
\end{tabular}

Table 2 Noise level of Gray cast iron stator shell motor

\begin{tabular}{|c|c|c|c|c|c|c|c|}
\hline \multicolumn{8}{|c|}{ Gray cast iron stator shell motor } \\
\hline \multirow{2}{*}{$2500 \mathrm{r} / \mathrm{min}$} & Frequency $(\mathrm{Hz})$ & 2832 & 2294.9 & 15136.7 & 1416 & 2002 & 14843.8 \\
\hline & Noise level(db) & 67.5 & 59.2 & 56.7 & 56.5 & 55.5 & 54.3 \\
\hline \multirow{2}{*}{$3500 \mathrm{r} / \mathrm{min}$} & Frequency $(\mathrm{Hz})$ & 2783.2 & 2441.4 & 2929.7 & 2636.7 & 14941.4 & 1416 \\
\hline & Noise level(db) & 71.8 & 62.2 & 61.6 & 58.7 & 58.5 & 55.5 \\
\hline \multirow{2}{*}{$4500 \mathrm{r} / \mathrm{min}$} & Frequency $(\mathrm{Hz})$ & 2734.4 & 2294.9 & 1367.2 & 537.1 & 683.6 & 1806.6 \\
\hline & Noise level(db) & 76.5 & 65.3 & 63.2 & 62.2 & 61.9 & 61.5 \\
\hline \multirow{2}{*}{$6000 \mathrm{r} / \mathrm{min}$} & Frequency $(\mathrm{Hz})$ & 3076.2 & 1220.7 & 2441.4 & 1855.5 & 97.7 & 2685.5 \\
\hline & Noise level(db) & 71 & 70 & 68.5 & 63.6 & 61.3 & 61.2 \\
\hline \multirow{2}{*}{$8000 \mathrm{r} / \mathrm{min}$} & Frequency $(\mathrm{Hz})$ & 2441.4 & 3222.7 & 146.5 & 830.1 & 1757.8 & 1611.3 \\
\hline & Noise level(db) & 76.4 & 72.1 & 71.7 & 71.2 & 69.6 & 68.9 \\
\hline \multirow{2}{*}{$9000 \mathrm{r} / \mathrm{min}$} & Frequency $(\mathrm{Hz})$ & 2685.5 & 1806.6 & 878.9 & 3613.3 & 146.5 & 8984.4 \\
\hline & Noise level(db) & 89.8 & 80.2 & 76.6 & 71.6 & 70.6 & 69.7 \\
\hline
\end{tabular}




\section{Interpretation of result}

Fig.3-Fig.5 gave the resonance frequency components and the acceleration, velocity of the vibration. It's no difficult to find that the resonance frequency components are all the harmonic frequencies of rotation rate of the motor. This is decided by the property of the electromagnetic force. Theoretically, the harmonic frequencies of electromagnetic force are infinite, it's impossible to avoid resonance of all this frequencies seemingly. In fact, as shown at the velocity frequency-domain curves in table 2 and table 3, only the frontal five frequencies are main components, other components approach zero. So, if we want to avoid resonance to reduce noise, it's only to avoid the natural frequency equaling to the frontal five frequencies, but it's still very difficult to avoid the five frequencies simultaneously. According to table 2 and table 3, the noise level is increase as the acceleration of the motor. For the aluminum stator shell motor, when the speed is $2500 \mathrm{r} / \mathrm{min}$, the noise level is $63.5 \mathrm{db}$, correspondingly, the frequency is $1364.1 \mathrm{~Hz}$. When the speed is $9000 \mathrm{r} / \mathrm{min}$, the noise level is $88.1 \mathrm{db}$, correspondingly, the frequency is $1806.6 \mathrm{~Hz}$. For the Gray cast iron stator shell motor, when the speed is $2500 \mathrm{r} / \mathrm{min}$, the noise level is $67.5 \mathrm{db}$, correspondingly, the frequency is $2832 \mathrm{~Hz}$. when the speed is $9000 \mathrm{r} / \mathrm{min}$, the noise level is $89.8 \mathrm{db}$, correspondingly, the frequency is $2685.5 \mathrm{~Hz}$. Although the structural style is similar and the speed is same, the vibration state is different. For the aluminum stator shell motor, the resonance frequencies distributed from $1074.2 \mathrm{~Hz}$ to $2539 \mathrm{~Hz}$. And the resonance frequencies of the gray cast iron stator shell motor are centered on $2800 \mathrm{~Hz}$. Now we will pay attention to the most noise level as are listed on the third column in table 2 and table 3 . The frequencies according to the most noise level of the aluminum stator shell SRM are $1074.2 \mathrm{~Hz}, 1364.1 \mathrm{~Hz}, 1416 \mathrm{~Hz}, 1855.5 \mathrm{~Hz}, 2539 \mathrm{~Hz}$. Based on the finite element analysis results, the resonance frequency $1074.2 \mathrm{~Hz}$ is mostly corresponding to the modes as are given in Fig.3 or Fig.4, the natural frequencies are $955.8 \mathrm{~Hz}$ and $958.22 \mathrm{~Hz}$, which can be excited and produce resonance by the electromagnetic force with $1074.2 \mathrm{~Hz}$ frequency. the resonance frequency $1364.1 \mathrm{~Hz}$ and $1416 \mathrm{~Hz}$ are mostly corresponding to the modes as are given in Fig.5 and Fig.6, the natural frequencies are $1743 \mathrm{~Hz}$ and $1641 \mathrm{~Hz}$. If the resonance frequency $1074.2 \mathrm{~Hz}$ is corresponding to the modes as are given in Fig.2, it should appear in the gray cast iron stator shell motor for they have the same rotors and speeds. However, the frequencies of the most noise level of the gray cast iron stator shell motor are $2400 \mathrm{~Hz}$ and $2800 \mathrm{~Hz}$, which corresponding to the mode given in Fig.6 and Fig.7. Therefore, the mode given in Fig.2 is not excited. The mode with frequency of $1074 \mathrm{~Hz}$ should be given in Fig.3. if we want to eliminate this noise, we should modify the structural style of the cover, this will change it's natural frequency. Other noises will be eliminated according to the same method.

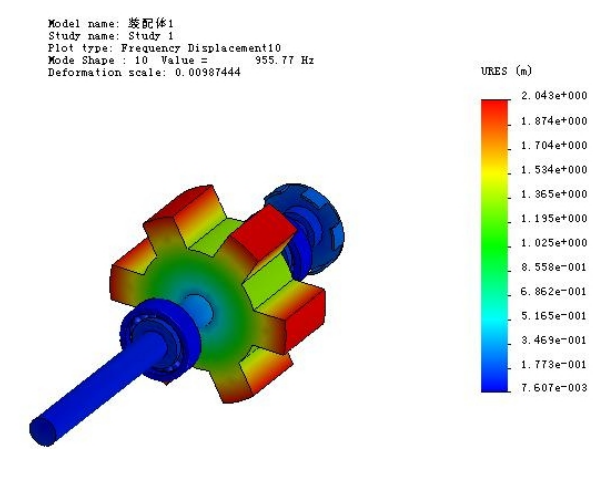

Fig.2 natural frequency of $\operatorname{rotor}(955.8 \mathrm{~Hz})$

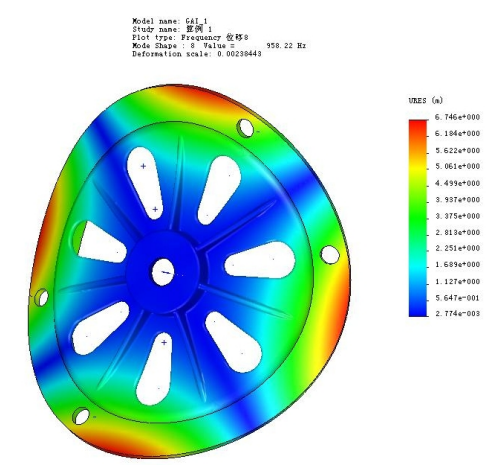

Fig.3 natural frequency of cover $(958.22 \mathrm{~Hz})$ 


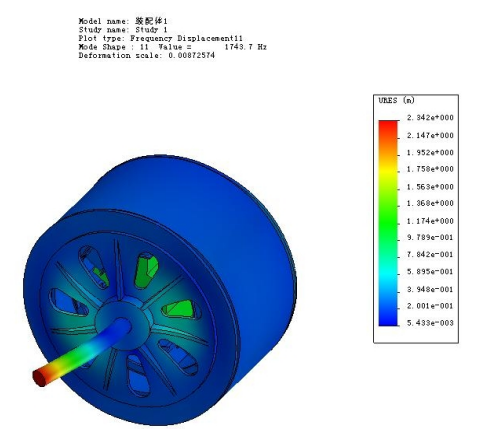

Fig.4 natural frequency of $\operatorname{motor}(1743 \mathrm{~Hz})$

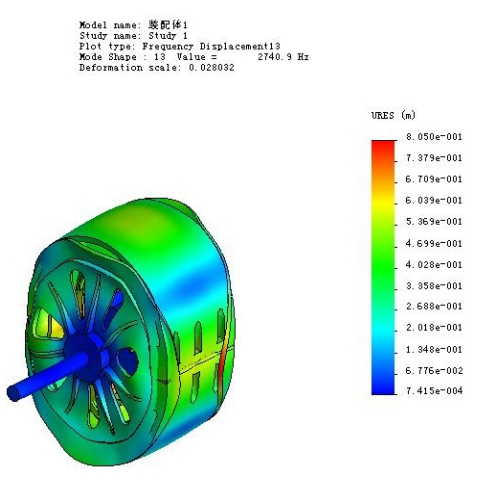

Fig.6 natural frequency of gray cast iron stator shell SRM $(2740.9 \mathrm{~Hz})$

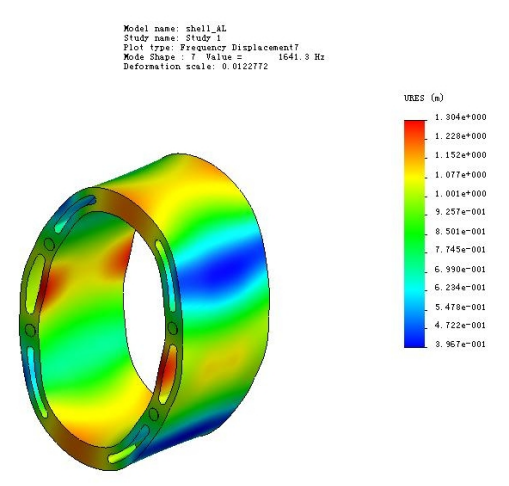

Fig.5 natural frequency of aluminum stator shell $(1641 \mathrm{~Hz})$

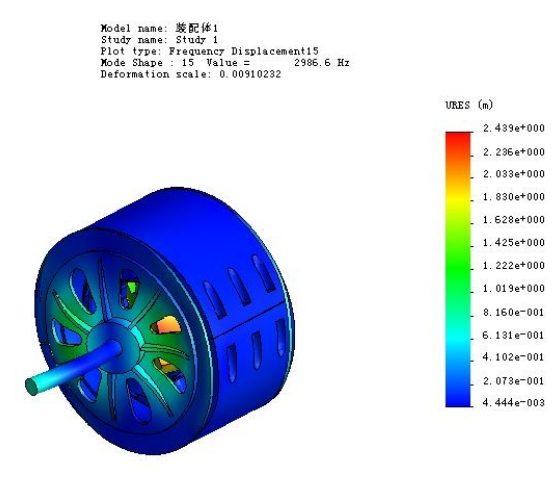

Fig.7 natural frequency of Gray cast iron stator shell SRM $(2986.6 \mathrm{~Hz})$

\section{Conclusion}

1、Based on the test results, the velocity frequency-domain curve shown that the harmonic frequency components which play a dominant role are the frontal five harmonic frequency components of the electromagnetic force. In order to avoid resonance, they will be mainly considered. All natural frequencies of the motor, include all parts, should be designed to avoid this harmonic frequency components.

2、 Compared with the gray cast iron stator shell motor, it's more difficult to control the noise of the aluminum stator shell motor. This is caused by the assembly relation between the housing case and the stator. For the aluminum stator shell motor, they were clearance fit. Because of the clearance, the housing case and the stator can not deform together under electromagnetic force, the resonance may occurs on the housing case, the stator or the cover. For the gray cast iron stator shell motor, the resonance occurs on the whole motor, because the housing case and the stator fitted tightly and the motor seem a whole. The noise of this structural style is more easily to be controlled.

3、The natural frequency of the cover is smaller than the stator. It's more possible to be excited and produce resonance. We should redesign the cover's structural style to enhance the natural frequency.

4、 The natural frequencies between $1000 \mathrm{~Hz}$ to $3000 \mathrm{~Hz}$ should be avoided in the motor structure design. 


\section{References}

[1] D. E. Cameron, J. H. Lang, and S. D.Umans, "The origin and reduction of acoustic noise in doubly salient variable-reluctance motors,” IEE Trans. Ind. Applicat., 1992, pp. 1250-1255.

[2] C. Pollock, and C. Y. Wu, "Acoustic noise cancellation technique for switched reluctance drives,'IEEE Trans. Ind. Applicat., Mar./Apr. 1997, pp. 477-484,.

[3] B. Fahimi, G. Suresh, K. M. Rahman, and M. Ehsani, "Mitigation of acoustic noise and vibration in switched reluctance motor drive using neural network based current profiling,"in Conf. Rec. IEEE-ISA Annu Meeting,. 1998, pp.715-722. 